\title{
Lifestyle-Related Factors in the Self-Management of Chemotherapy-Induced Peripheral Neuropathy in Colorectal Cancer: A Systematic Review
}

\author{
Tess M. E. Derksen, ${ }^{1}$ Martijn J. L. Bours, ${ }^{1}$ Floortje Mols, ${ }^{2,3}$ and Matty P. Weijenberg ${ }^{1}$ \\ ${ }^{1}$ Department of Epidemiology, School for Oncology and Developmental Biology (GROW), Maastricht University, \\ Maastricht, Netherlands \\ ${ }^{2}$ Department of Medical and Clinical Psychology, Tilburg University, Tilburg, Netherlands \\ ${ }^{3}$ Netherlands Comprehensive Cancer Organization, Netherlands Cancer Registry, Eindhoven, Netherlands \\ Correspondence should be addressed to Martijn J. L. Bours; m.bours@maastrichtuniversity.nl
}

Received 15 December 2016; Revised 24 February 2017; Accepted 1 March 2017; Published 16 March 2017

Academic Editor: Kenji Watanabe

Copyright $\odot 2017$ Tess M. E. Derksen et al. This is an open access article distributed under the Creative Commons Attribution License, which permits unrestricted use, distribution, and reproduction in any medium, provided the original work is properly cited.

\begin{abstract}
Background. Chemotherapy-induced peripheral neuropathy (CIPN) is a common adverse effect of chemotherapy treatment in colorectal cancer (CRC), negatively affecting the daily functioning and quality of life of CRC patients. Currently, there are no established treatments to prevent or reduce CIPN. The purpose of this systematic review was to identify lifestyle-related factors that can aid in preventing or reducing CIPN, as such factors may promote self-management options for CRC patients suffering from CIPN. Methods. A literature search was conducted through PubMed, Embase, and Google Scholar. Original research articles investigating oxaliplatin-related CIPN in CRC were eligible for inclusion. Results. In total, 22 articles were included, which suggested that dietary supplements, such as antioxidants and herbal extracts, as well as physical exercise and complementary therapies, such as acupuncture, may have beneficial effects on preventing or reducing CIPN symptoms. However, many of the reviewed articles presented various limitations, including small sample sizes and heterogeneity in study design and measurements of CIPN. Conclusions. No strong conclusions can be drawn regarding the role of lifestyle-related factors in the management of CIPN in CRC patients. Certain dietary supplements and physical exercise may be beneficial for the management of CIPN, but further research is warranted.
\end{abstract}

\section{Introduction}

Colorectal cancer (CRC) is a highly prevalent type of cancer and a common cancer-related cause of death worldwide [1]. In the past decades, an increase in survival and prevalence rates has resulted from the development of novel treatments and improved diagnostic methods, including early detection strategies through the implementation of population screening programmes. Consequently, more people nowadays survive longer after a CRC diagnosis and live with the physical and mental consequences of the disease and the adverse effects of its treatment [2-4].

CRC is treated through surgery in most cases, combined with (neo)adjuvant chemotherapy and/or radiotherapy depending on the cancer stage [5]. It is known that chemotherapy can cause severe adverse effects that can be highly detrimental to a patient both physiologically and psychologically, thus having a large impact on an individual's quality of life [6]. One common and severe adverse effect of chemotherapy is chemotherapy-induced peripheral neuropathy (CIPN), which usually consists of sensory axonopathies [6]. Although it can depend on the specific affected site of the peripheral nervous system, common signs and symptoms of CIPN usually include numbness and tingling of hands/feet, muscle weakness, lancinating/burning pain, cutaneous hyperesthesia, and loss of pain/temperature sensation [6]. CIPN may develop weeks to months after exposure to chemotherapeutic agents, can continue in spite of cessation of chemotherapy, and may be irreversible depending on the degree of neuron damage [6]. The chemotherapeutic agents 
which most frequently cause CIPN are thalidomide, bortezomib, platinum derivatives, vinca alkaloids, and taxanes [7].

Platinum-based chemotherapy is the standard treatment for stage III-IV CRC, and it may also be recommended to high-risk stage II patients $[5,8,9]$. Oxaliplatin, a cytotoxic platinum compound which has been used in Europe since 1996, is one of the chemotherapeutic agents used in CRC [10]. Around $60 \%$ of CRC patients will receive oxaliplatin-based chemotherapy as part of their cancer treatment. Approximately $48 \%$ of patients receiving oxaliplatin-based chemotherapy will develop neuropathy symptoms during treatment, which may resolve after treatment or develop into chronic symptoms in $20-50 \%$ of these patients (a phenomenon known as "coasting") [11, 12]. Another chemotherapeutic agent is cisplatin [13]. Platinum compounds form so-called platinum complexes which inhibit DNA replication, thus leading to apoptosis [7, 14]. It is believed that acute oxaliplatin-related CIPN may be the result of rapid chelation of calcium by oxaliplatin-induced oxalate and that oxaliplatin is able to alter voltage-gated sodium channels, most likely through a pathway involving calcium ions [14]. Another mechanism that has been postulated to contribute to the development of CIPN is a decrease in cellular metabolism in dorsal root ganglion cells [14]. Possible risk factors associated with oxaliplatin-related CIPN include dosage, cumulative dose, treatment schedule, and the patient's muscle mass $[14,15]$.

An increasing number of CRC survivors suffer from the negative and disabling consequences that CIPN has on their quality of life [3]. Currently, there are no effective treatment options for preventing CIPN or decreasing its symptoms. Neuroprotective agents such as thiols, neurotrophic factors, and anticonvulsants have been tested as possible preventive agents, but no conclusive results have been published [16]. For this reason, research is now also focusing on the potential of lifestyle factors for reducing or preventing CIPN [3]. These lifestyle factors refer to health-related behaviors, such as physical exercise, dietary habits, and intake of dietary supplements, as well as the use of natural products or complementary therapies which patients choose to engage in to selfmanage their CIPN symptoms, without necessary physician supervision. Previous research has suggested that lifestyle factors, such as an unhealthy diet and body composition, play an important role in CRC development and also affect the health-related quality of life of CRC survivors $[1,17]$. For this reason, it is of interest to investigate whether such lifestyle factors could also play a role in the development and consequences of CIPN in CRC survivors.

Although a recent systematic review by Brami et al. [18] reported evidence from randomized clinical trials (RCTs) on effects of natural products and complementary therapies on CIPN in general, the focus of the present systematic review was to evaluate available evidence from research on lifestylerelated factors and their effects specifically on oxaliplatinrelated CIPN in CRC. Therefore, the aim of this systematic literature review was to identify lifestyle factors that play a role in the development and occurrence of CIPN symptoms. Such factors could provide avenues for reducing the occurrence of CIPN in CRC patients as well as for promoting self-management options for CRC survivors suffering from chronic CIPN symptoms.

\section{Methods}

For the purpose of this review, a systematic search of the literature was conducted through PubMed, Embase, and Google Scholar. The initial literature search, limited to the time period between 1994 and 2015, was performed on December 8, 2015, by the use of the following search strategy: ((colon OR rectum OR colorectal) AND (cancer OR neoplasm) AND (neuropathy OR peripheral neuropathy) AND (chemotherapy OR platin) AND (lifestyle OR behavior* OR management OR supplement ${ }^{*}$ OR vitamin* OR mineral* OR alternative therapy OR complementary therapy OR BMI OR exercise OR physical activity)).

2.1. Article Selection. A total of 1,077 records were identified in the initial search (Figure 1). Only original research articles describing the prevention and/or management of oxaliplatinrelated CIPN in colon, rectum, or CRC patients were eligible for inclusion. All types of study designs were eligible, except for case reports because of the low level of evidence provided by these studies. For studies containing mixed cancer populations that included CRC, the results of CIPN among CRC patients had to be reported separately from the results of CIPN among patients with other tumors to be eligible for inclusion. Furthermore, articles were required to describe lifestyle-related or alternative/complementary management options for CIPN. For this reason, studies describing prescription medication were not eligible. Studies in which dietary supplements were administered intravenously were also ineligible, and so were animal or cell studies. Finally, studies were only included if the publication was an original research article (e.g., no systematic reviews, book chapters, dissertations, poster abstracts, editorials, and letters to the editor) and if the study had been published in a peer-reviewed journal in English. On the basis of these criteria, 17 full-text articles deriving from the initial search were evaluated for eligibility. An additional 25 articles were identified through citation tracking and screened for eligibility (Figure 1).

\section{Results}

3.1. Study Characteristics. A total of 22 articles were included in the review, all of which were published between 1994 and 2015 (see Table 1 for a summary of study characteristics and main findings per article) $[3,11,19-38]$. The majority of the articles reviewed reported results of randomized trials $(N=11)[19-21,23,24,26-28,31-33]$. Other research designs included nonrandomized trials $(N=5)$ [22, 34-37], crosssectional studies $(N=2)[3,11]$, cohort studies $(N=2)$ $[25,29]$, case series $(N=1)[38]$, and a crossover trial $(N=$ 1) [30]. Furthermore, the study populations were comprised of (exclusively) colon cancer or CRC patients $(N=15)$ $[3,11,21,22,24-29,31-34,38]$ and mixed cancer patients, including CRC $(N=7)[19,20,23,30,35-37]$. 


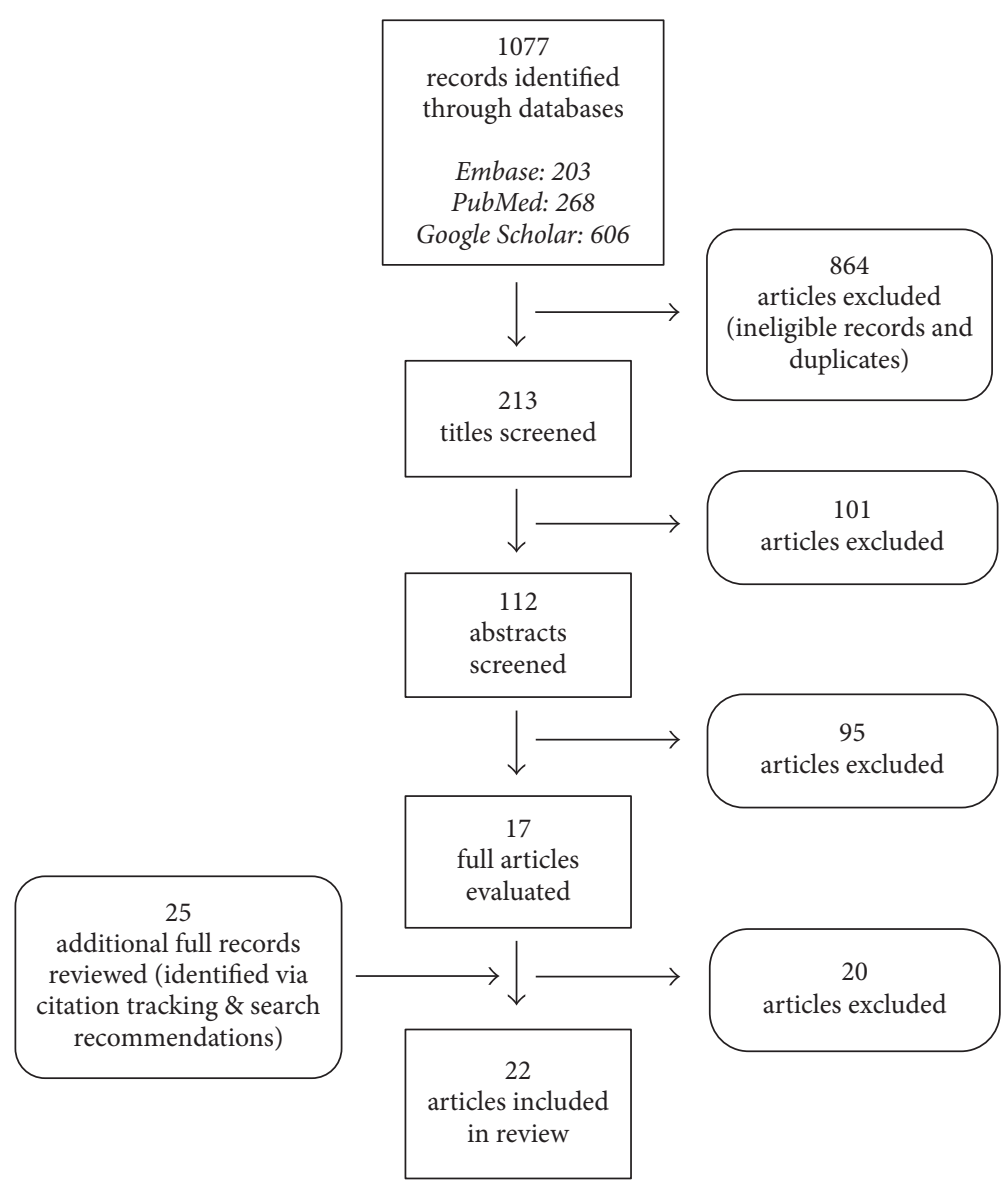

FIgURE 1: Flow chart showing results of literature search and article screening and selection.

3.2. Measures of Chemotherapy-Induced Peripheral Neuropathy (CIPN). Overall, the articles presented a wide variety of measures to assess CIPN. Six studies made use of the National Central Cancer Institute Common Terminology Criteria for Adverse Events (NCI-CTCAE v.3 or v.4) [19, 20, 26, 29, 36, 38]. Four studies graded CIPN according to the National Cancer Institute Common Toxicity Criteria (NCI-CTC) [24, $28,31,33]$, two other studies applied the World Health Organization Toxicity Criteria [30,32], and further three studies implemented the Neurotoxicity Criteria of Debiopharm (DEB-NTC) $[25,27,28]$. One study used a symptom experience diary questionnaire for peripheral neuropathy assessment [21]. Moreover, two studies used the Functional Assessment of Cancer Therapy/Gynecologic Oncology GroupNeurotoxicity (FACT/GOG-Ntx) questionnaire [23, 36], two other studies used the Chemotherapy-Induced Peripheral Neuropathy Assessment Tool (CIPNAT) [11, 34], and another two studies used the European Organisation for Research and Treatment of Cancer Quality of Life Questionnaire, Chemotherapy-Induced Peripheral Neuropathy scale (EORTC QLQ-CIPN20) [3, 35]. Another method to evaluate CIPN included measurements of nerve conduction velocity [37]. Finally, one study did not specify the CIPN assessment measures that had been used [22].
3.3. Lifestyle Factors. Results are presented in Table 1. The lifestyle factors identified through the literature search were grouped into four categories: (I) dietary supplements, (II) physical activity, (III) alternative (complementary) therapies, and (IV) multiple/other strategies.

(I) Dietary Supplements. The most researched natural remedy for CIPN was Goshajinkigan (GJG or TJ-107), a traditional Japanese herbal extract ("Kampo formula"). Four included studies evaluated the effects of oral administration of GJG and concluded that administering a minimum of 2.5 grams of GJG during chemotherapy significantly reduced the toxic effects of oxaliplatin, reducing the incidence of CIPN and its intensity [25-27, 29]. More specifically, Kono et al. [25] found that the incidence of CIPN symptoms at higher doses of oxaliplatin was lower in the groups receiving GJG. At the same time, patients receiving GJG tolerated higher cumulative doses of oxaliplatin [25]. A study by Nishioka et al. [27] found a significant reduction of grade III CIPN for patients receiving GJG. Finally, Yoshida et al. [29] observed a significant difference in the development of CIPN between GJG and control patients, with less than 10\% of GJG patients presenting CIPN symptoms. In contrast, however, a recent randomized placebo-controlled trial by Oki et al. [28] showed 


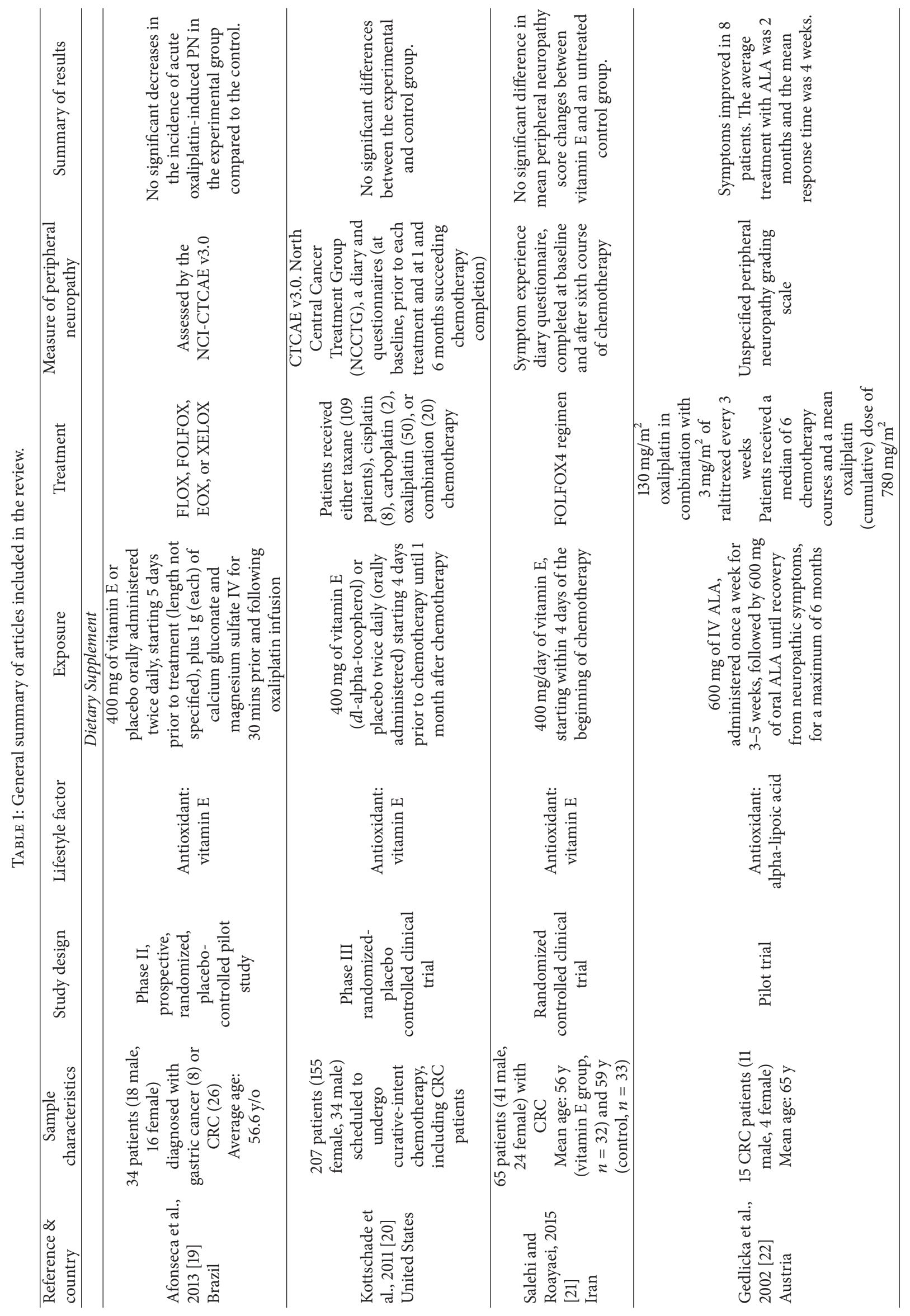




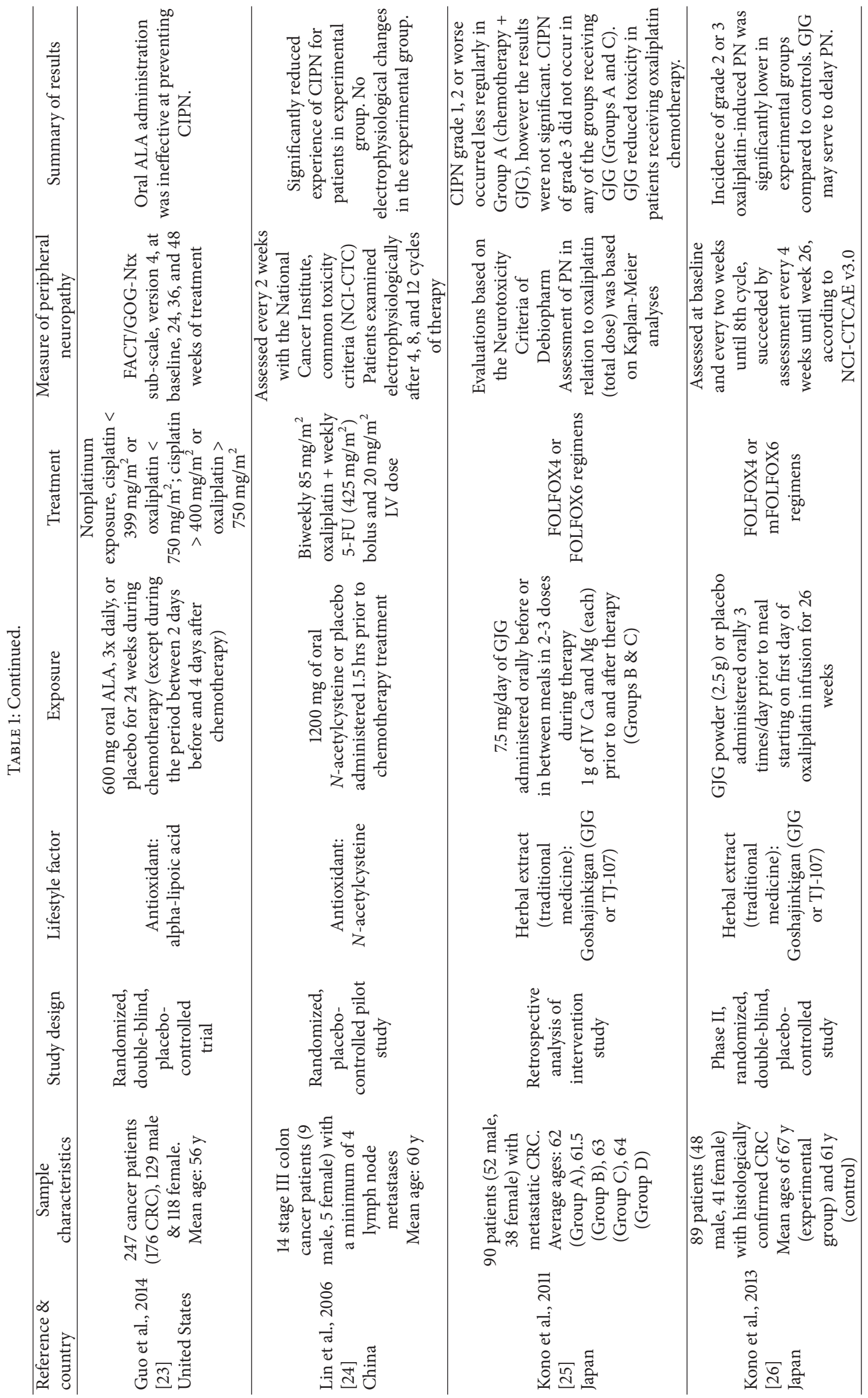




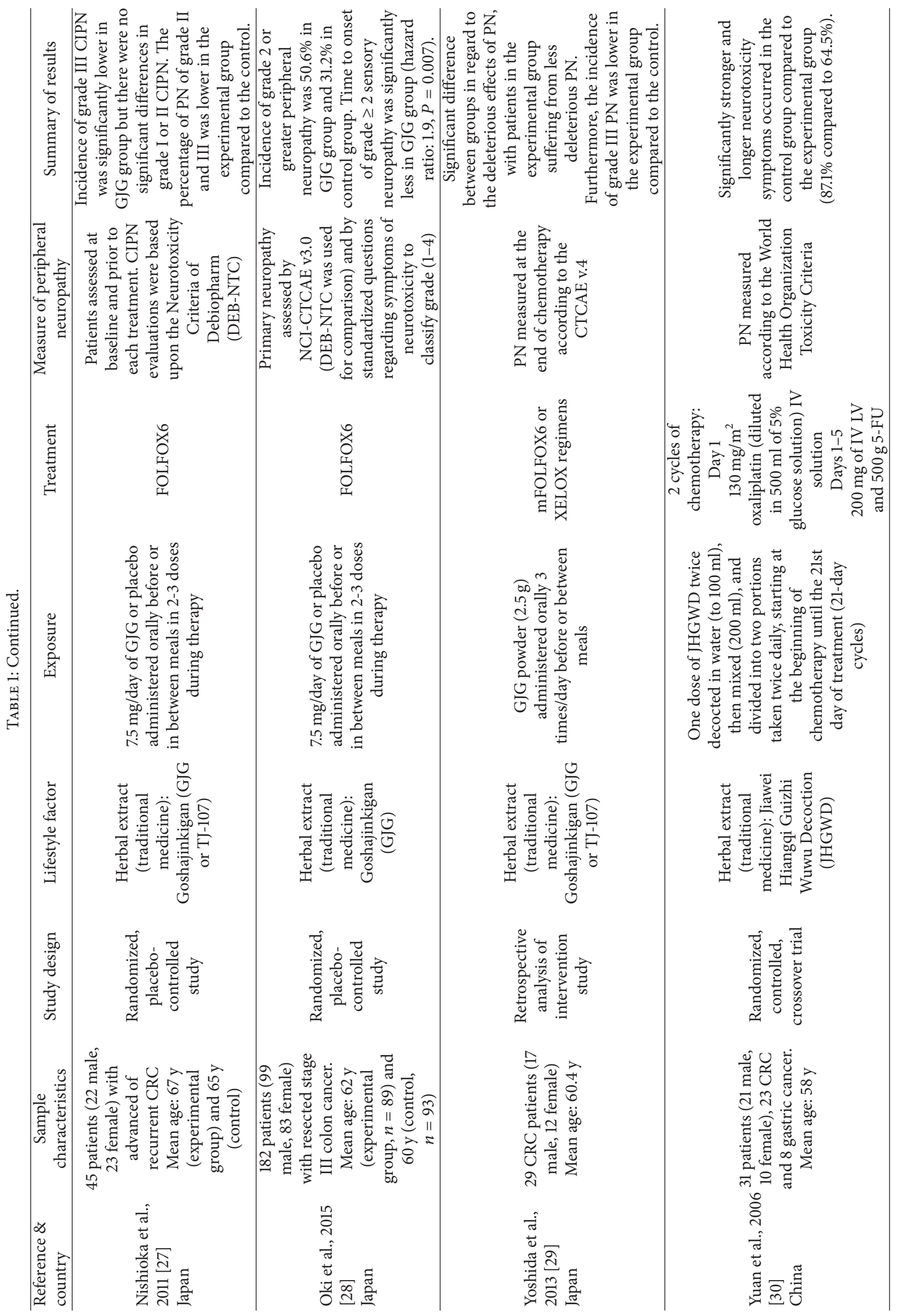




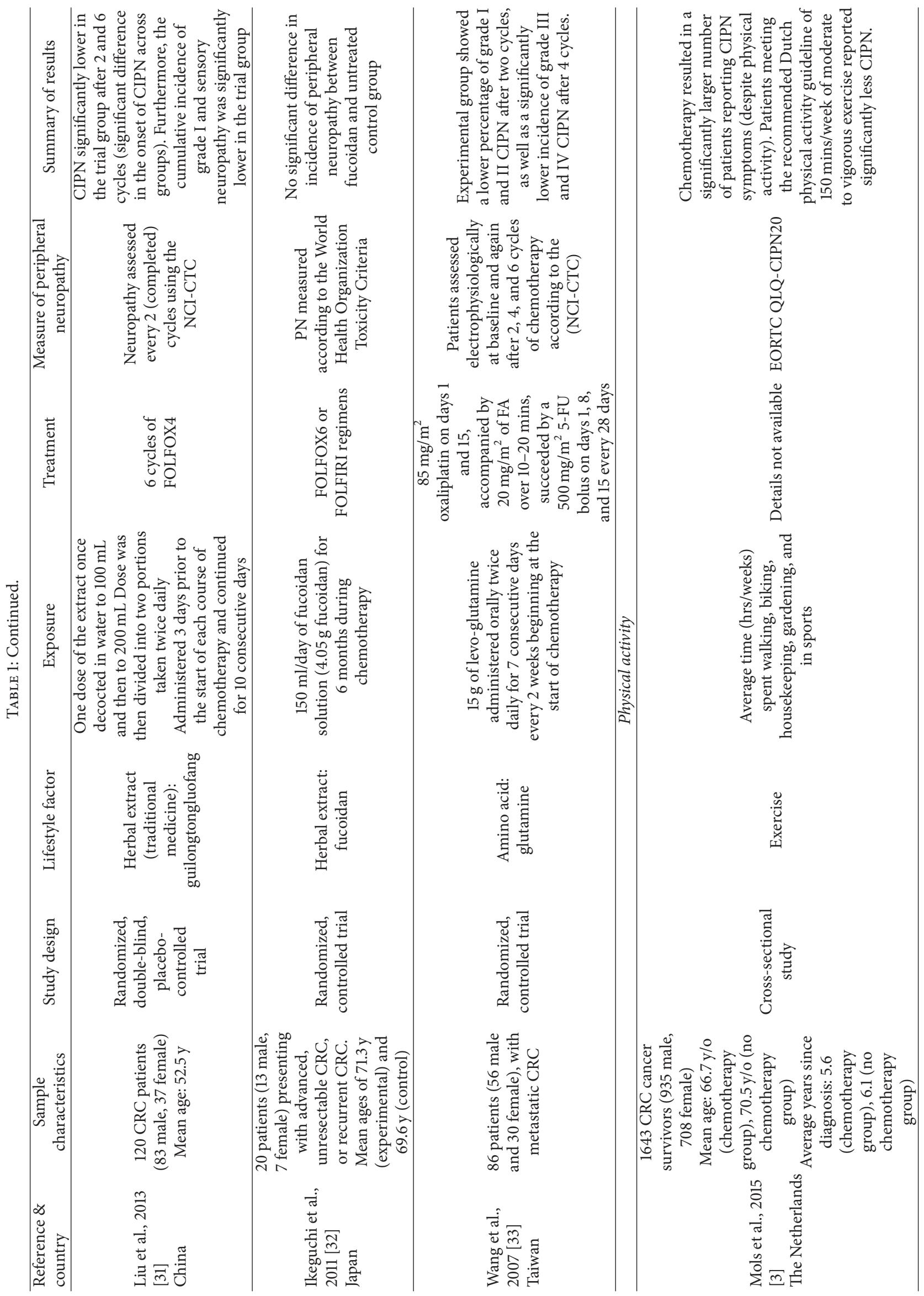




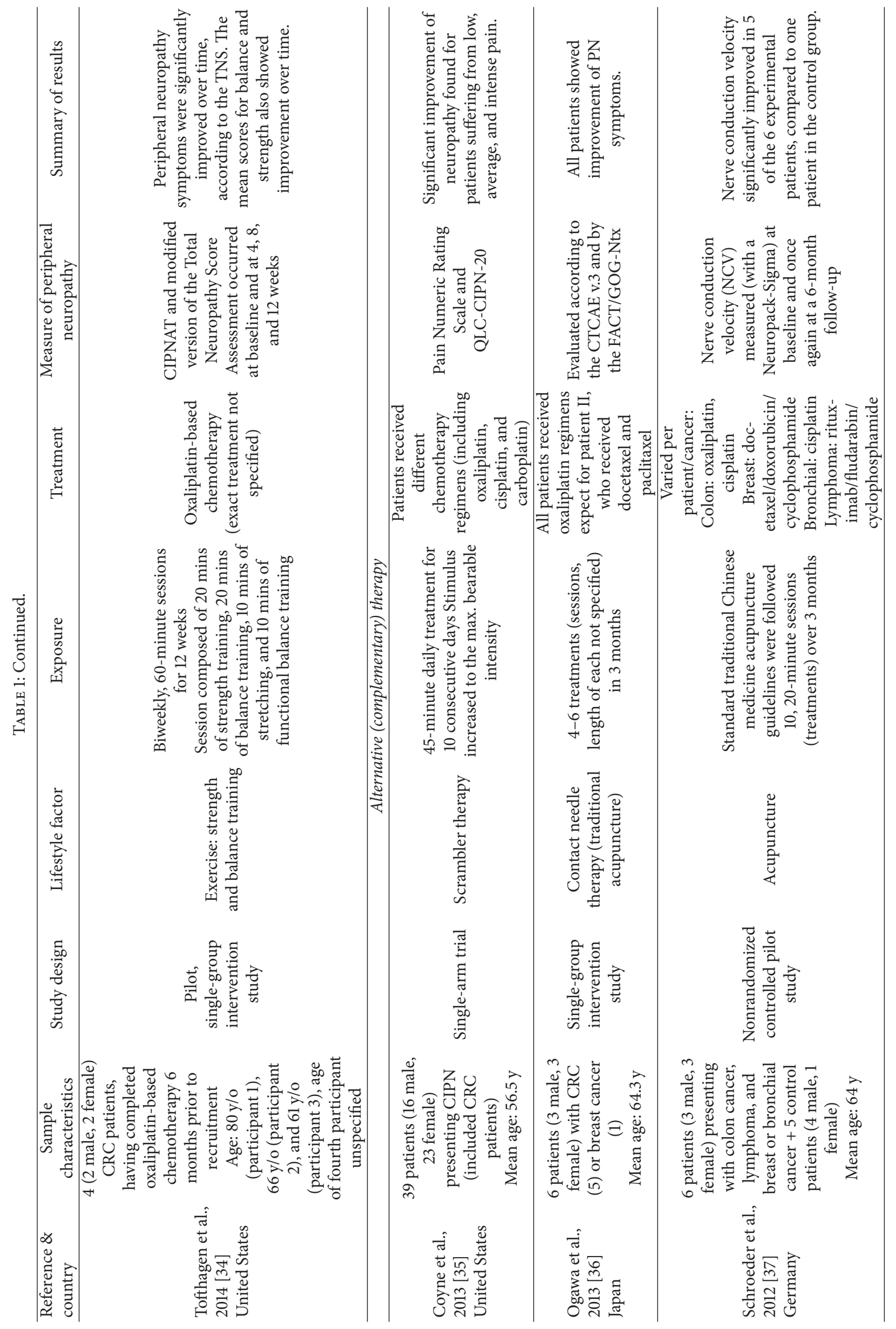




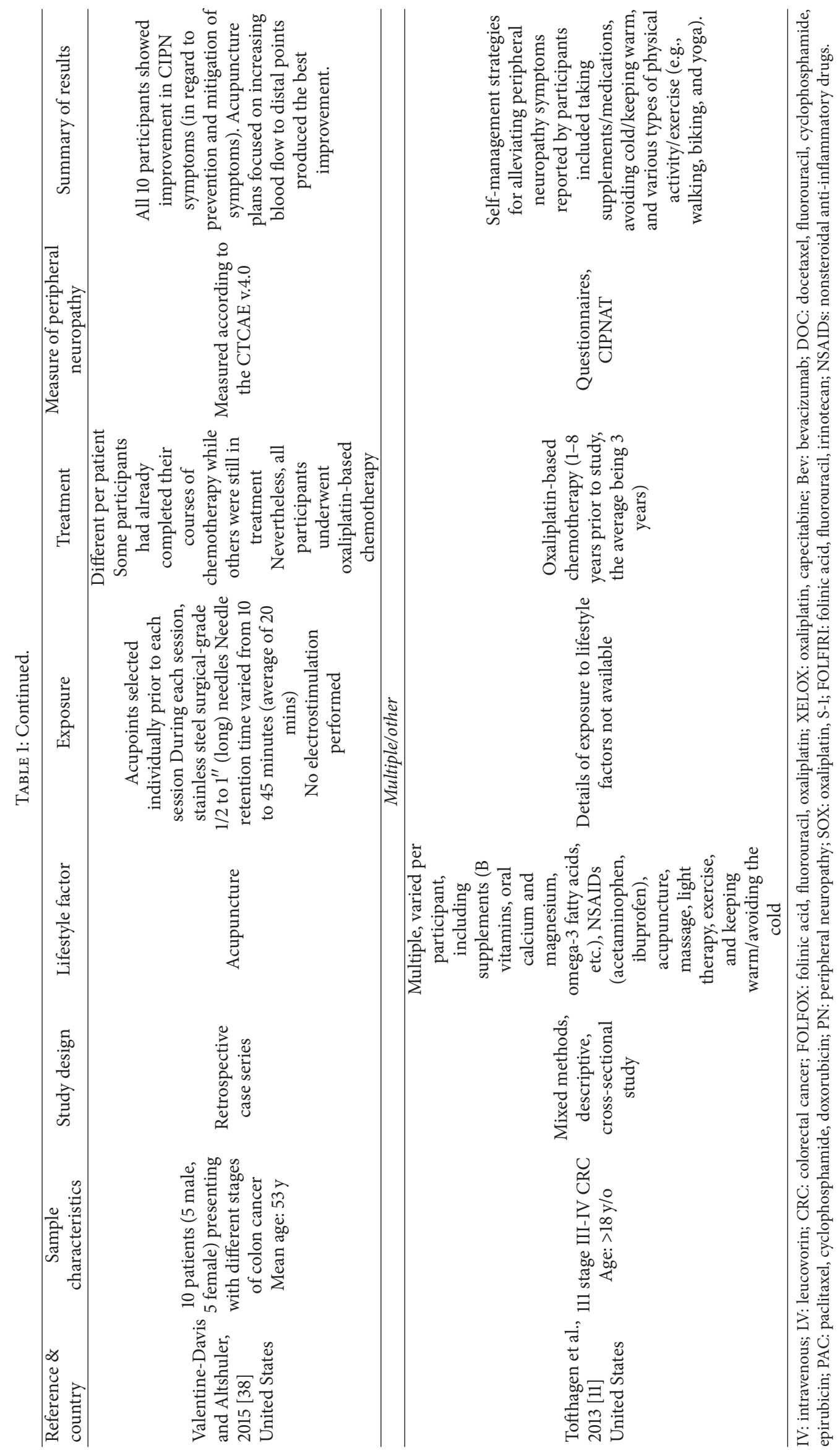


that GJG supplementation $(7.5 \mathrm{mg} / \mathrm{d})$ during FOLFOX6 chemotherapy in patients with resected stage III colon cancer led to a significantly earlier time to onset of sensory neuropathy (grade 2 or greater) compared to a placebo group.

A further traditional herbal extract that appeared to have a positive effect on CIPN was the Jianwei Hiangqi Guizhi Wuwu Decoction (JHGWD), a traditional Chinese medicine composed of botanical materials [30]. Administration of JHGWD during oxaliplatin chemotherapy significantly reduced the intensity of CIPN symptoms, as well as their duration [30]. Furthermore, the symptoms presented by control patients were more severe in nature and of longer duration [30]. Another traditional Chinese medicinal remedy studied was guilongtongluofang, an aqueous extract of dried herbs. In their study, Liu et al. [31] found that $200 \mathrm{~mL}$ of guilongtongluofang taken twice daily significantly lowered the percentages of grade I and II CIPN after 2 and 6 cycles of chemotherapy. The risk of grade I CIPN was significantly lower in the treatment group, and the onset of CIPN symptoms was significantly later in the treatment group than in the control group (9.4 weeks versus 6.5 weeks, resp.) [31]. Furthermore, a randomized controlled study of fucoidan, a sulfated polysaccharide extracted from brown seaweed, showed that the incidence of peripheral neuropathy in patients with advanced CRC was not significantly different between a fucoidan-treated group and an untreated control group [32].

Three studies investigated the effect of vitamin E (400 mg) on CIPN and found no significant differences between oral administration of vitamin $E$ and placebo $[19,20]$ or an untreated control group [21]. Contradicting results were found in relation to the effects of the antioxidant alpha-lipoid acid (ALA). In a study by Gedlicka et al. [22], 7 patients suffering from grade II CIPN and 1 patient presenting grade III CIPN showed improvement of at least one grade. In contrast, Guo et al. [23] found no significant differences between ALA and placebo. Another antioxidant, $N$-acetylcysteine, was shown to have beneficial effects in patients undergoing oxaliplatin chemotherapy by reducing the incidence of CIPN, as well as by reducing neurophysiological changes during the course of treatment [24].

Finally, supplementation of the amino acid glutamine in patients undergoing oxaliplatin chemotherapy for metastatic CRC resulted in reduction of grade I and II CIPN following two cycles of therapy and reduction of grade III and IV CIPN after four and six cycles [33]. Patients also reported less CIPNrelated interference of their daily activities (7.1\% interference for patients receiving glutamine versus $40.9 \%$ interference for control patients) [33].

(II) Physical Activity. Evidence supporting a role of physical activity in alleviating CIPN symptoms was scarce; only two studies were identified. According to a cross-sectional study by Mols et al. [3], CRC survivors who reported to meet the recommended Dutch physical activity guideline of 150 minutes of moderate-to-vigorous physical activity per week also reported less CIPN symptoms. Furthermore, a small single-arm trial on the effectiveness of strength and balance training for CRC patients, who had finished their chemotherapy treatment, showed improved balance and lower extremity strength after four weeks of biweekly 60-minute training sessions, as well as improved neuropathic symptoms [34].

(III) Alternative (Complementary) Therapies. Some evidence was found for the use of complementary therapies to manage neuropathic symptoms, such as acupuncture. A small pilot study by Ogawa et al. [36] reported improved neuropathic symptoms after 4-6 sessions of contact needle therapy (traditional acupuncture). Another small study observed that acupuncture improved nerve conduction velocity in mixed cancer patients, including CRC, with CIPN [37]. Similarly, a case series of 10 patients receiving traditional Chinese acupuncture showed significant amelioration of CIPN symptoms, as well as lowered incidence of CIPN and reduction of symptom mitigation [38]. A further study tested the effects of Scrambler therapy in patients with CIPN, a method of cutaneous nerve stimulation that aims to relieve pain by providing "nonpain" information to block the action of pain stimuli [35]. Statistically significant improvements were observed in pain perception, motor and sensory scales, and interference with life scores [35].

(IV) Multiple/Other Strategies. A single cross-sectional study was identified which described multiple self-management strategies applied by stage III-IV CRC patients in order to minimize neuropathic symptoms [11]. A variety of strategies were mentioned by participants, including use of dietary supplements (e.g., B vitamins, ALA, and glutamine), over-thecounter medications (e.g., nonsteroidal anti-inflammatory drugs), and nonpharmacological strategies such as avoiding cold/keeping warm, massaging/rubbing affected areas, and various types of physical activity or exercise (e.g., walking, biking, or yoga) [11]. However, since only qualitative data analyses were performed in this study, no significant relief effects of the reported self-management strategies could be shown.

\section{Discussion}

Currently, there are no established treatments for CIPN. This systematic literature review aimed to identify lifestylerelated factors that could potentially empower CRC patients to decrease the risk of developing CIPN and/or reduce CIPN symptoms, thereby reducing the impact of CIPN on their daily functioning and quality of life. Although 22 eligible studies were identified that described a number of lifestyle-related factors, no conclusive evidence was provided regarding any particular factor. Nevertheless, the findings of this review may offer clues for potentially relevant lifestyle factors that could be further explored in future studies.

There is some weak evidence pointing to beneficial effects of herbal extracts or supplements for managing or preventing CIPN in CRC. In general, most evidence points to traditional medicines, such as GJG, as possible management options for CIPN symptoms [25-27, 29]. This evidence is further supported by animal studies suggesting that GJG may prevent cold hyperalgesia and mechanical allodynia without adversely affecting the antitumor effect of oxaliplatin on tumor cells [39]. However, one study also showed negative effects of GJG 
on neuropathy [28] and the majority of studies reviewed were limited by design, such as having small sample sizes, and results were difficult to compare because of the use of different methods to measure CIPN. Therefore, the results of these studies should be interpreted with caution. Regarding the discrepancy across articles with regard to the method of measuring CIPN, it is important to note that toxicity scales can vary in the way they score symptoms. A change from "grade II to grade I" CIPN defined according to one scale may not be directly comparable to another study in which a change in CIPN is defined based on a different scale. For this reason, varying CIPN measurement methods may therefore explain why contradictory results have been observed in different studies on the same variable. This could, for instance, have been the case for the contradicting results regarding the effect of ALA on CIPN [22, 23]. Furthermore, although questionnaires and self-reports are the preferred measure of CIPN [16], they can be highly susceptible to social desirability bias and be influenced by the ability of participants to accurately recall and estimate the level of past pain symptoms.

Besides the variation in measurement methods, there was a large variation in sample sizes of the reviewed studies. The largest study included 1,644 CRC survivors [3], while 7 out of the 22 articles reviewed reported findings of studies with less than 20 participants [22, 24, 32, 34, 36-38]. As it is hardly possible to generalize the results obtained in studies with such small sample sizes, future studies in larger samples of CRC patients are needed. Furthermore, 7 articles reported results of studies in mixed cancer samples $[19,20,23,30,35-$ 37]. Although these mixed cancer populations were included in this review since they also contained CRC patients, it is possible that the results of these studies reflected effects of the lifestyle factor of interest on CIPN in patients with cancers other than CRC. The reviewed articles also presented a large variety in study designs. Only 11 out of the 22 articles described results of randomized controlled trials, which would be the preferred design for evaluating effects of lifestyle factors on CIPN.

Though the evidence for a role of lifestyle-related factors in preventing and managing CIPN was weak in general, the results of this review may provide relevant leads for future research. Some areas of further research have already been mentioned, and a few others can be pointed out; especially some of the self-management methods described by patients themselves may warrant further investigation [11]. Factors of interest could be, for example, the use of NSAIDs or B vitamins to alleviate chronic CIPN symptoms. Other studies on CIPN, which did not meet the inclusion criteria for the present review, mentioned meditation, yoga, and Reiki [40], as well as gingko biloba and acetyl-L-carnitine supplementation [41, 42], as possibly relevant lifestyle-related factors. Furthermore, although the studies reviewed did not find any relationship between vitamin E and CIPN in CRC patients treated with oxaliplatin, studies testing the efficacy of this micronutrient to reduce CIPN in cisplatin (a platinum compound similar to oxaliplatin) therapy have shown beneficial effects [43-45]. The relationship between vitamin $\mathrm{E}$ and CIPN should thus be studied further. In addition, the study by Lin et al. [24] offers a potentially promising treatment option warranting further investigation, as $\mathrm{N}$-acetylcysteine naturally increases blood levels of glutathione, which is another antioxidant that has also been shown to possess neuroprotective qualities when administered intravenously during oxaliplatin therapy [46, 47]. Moreover, the evidence that glutamine could help manage CIPN has been further supported by animal studies and intravenous administration of the amino acid $[48,49]$. Finally, it might be interesting to investigate the role of calcium and magnesium supplementation, as suggested by the findings of a case report [50] and also because some evidence exists regarding the role of calcium and magnesium infusions on platinum-related CIPN [51-54].

Somewhat unexpectedly, evidence regarding the role of physical activity on CIPN was found to be limited in CRC survivors. There is evidence that physical activity reduces CIPN in breast cancer patients, ovarian cancer patients, and patients with diabetic peripheral neuropathy [55-59]. For this reason, further studies in CRC survivors are highly needed to examine the effects of physical activity on CIPN, especially since physical activity can have beneficial effects on many health-related outcomes in CRC survivors. Moreover, although the evidence concerning CRC is limited, evidence suggests that acupuncture can reduce the symptoms of nonCRC CIPN, diabetic peripheral neuropathy, and neuropathic symptoms experienced by HIV-positive patients [60-63]. It could therefore be relevant to further investigate acupuncture treatments, as such traditional medicine might offer patients a drug-free coping mechanism for neuropathic pain. Lastly, animal studies have provided insight into other factors/ supplements which could be worth studying in CRC patients, such as curcumin, flavonoids, reduced poly-amine diets, and vitamin $\mathrm{C}$, all of which have been found to decrease CIPN in mice models [64-68].

\section{Conclusion}

The results of this systematic review indicate that evidence regarding lifestyle-related factors and their potential for the prevention and management of CIPN is very limited. The studies identified in this review might suggest a possible role for supplements such as herbal extracts, physical activity, and alternative therapies such as acupuncture in the selfmanagement of CIPN. Further research with stronger study designs, larger samples, and uniform measures of CIPN is pivotal to confirm such claims.

Taken together, the studies reviewed are inconclusive regarding a possible role for lifestyle-related factors in the management of CIPN in CRC. Nevertheless, this systematic review can provide potential guidance for future studies concerning the role of lifestyle factors in the development and management of CIPN in CRC.

\section{Disclosure}

All authors have approved of the contents of the manuscript.

\section{Conflicts of Interest}

The authors report no conflicts of interest. 


\section{Authors' Contributions}

Tess M. E. Derksen and Martijn J. L. Bours contributed equally to this work.

\section{Acknowledgments}

Martijn J. L. Bours is supported by the Alpe d'HuZes Foundation within the research program "Leven met kanker" of the Dutch Cancer Society (Grant no. UM-2012-5653) and by Kankeronderzoekfonds Limburg as part of Health Foundation Limburg (Grant no. 00005739). This work was part of an internship from University College Utrecht performed by Tess M. E. Derksen in 2015 at the Department of Epidemiology, Faculty of Health, Medicine and Life Sciences, Maastricht University, The Netherlands.

\section{References}

[1] J. Ferlay, E. Steliarova-Foucher, J. Lortet-Tieulent et al., "Cancer incidence and mortality patterns in Europe: estimates for 40 countries in 2012," European Journal of Cancer, vol. 49, no. 6, pp. 1374-1403, 2013.

[2] Cijfersoverkanker.nl, Dutch Cancer Registry, 2015, Integraal Kankercentrum Nederland, http://www.cijfersoverkanker.nl.

[3] F. Mols, A. J. M. Beijers, G. Vreugdenhil, A. Verhulst, G. Schep, and O. Husson, "Chemotherapy-induced peripheral neuropathy, physical activity and health-related quality of life among colorectal cancer survivors from the PROFILES registry," Journal of Cancer Survivorship, vol. 9, no. 3, pp. 512-522, 2015.

[4] F. N. Van Erning, L. N. Van Steenbergen, V. E. P. P. Lemmens et al., "Conditional survival for long-term colorectal cancer survivors in the Netherlands: who do best?" European Journal of Cancer, vol. 50, no. 10, pp. 1731-1739, 2014.

[5] A. G. Pallis and I. A. Mouzas, "Adjuvant chemotherapy for colon cancer," Anticancer Research, vol. 26, no. 6, pp. 4809-4815, 2006.

[6] A. J. Ocean and L. T. Vahdat, "Chemotherapy-induced peripheral neuropathy: pathogenesis and emerging therapies," Supportive Care in Cancer, vol. 12, no. 9, pp. 619-625, 2004.

[7] W. Grisold, G. Cavaletti, and A. J. Windebank, "Peripheral neuropathies from chemotherapeutics and targeted agents: diagnosis, treatment, and prevention," Neuro-Oncology, vol. 14, supplement 4, pp. iv45-iv54, 2012.

[8] S. K. Kumar and R. M. Goldberg, "Adjuvant chemotherapy for colon cancer," Current Oncology Reports, vol. 3, no. 2, pp. 94-101, 2001.

[9] G. A. Poultsides, E. L. Servais, L. B. Saltz et al., "Outcome of primary tumor in patients with synchronous stage IV colorectal cancer receiving combination chemotherapy without surgery as initial treatment," Journal of Clinical Oncology, vol. 27, no. 20, pp. 3379-3384, 2009.

[10] J. Graham, M. Muhsin, and P. Kirkpatrick, "Oxaliplatin," Nature Reviews Drug Discovery, vol. 3, no. 1, pp. 11-12, 2004.

[11] C. Tofthagen, L. Gonzalez, C. Visovsky, and A. Akers, "Selfmanagement of oxaliplatin-related peripheral neuropathy in colorectal cancer survivors," Chemotherapy Research and Practice, vol. 2013, Article ID 547932, 7 pages, 2013.

[12] S. B. Park, C. S. Y. Lin, A. V. Krishnan, D. Goldstein, M. L. Friedlander, and M. C. Kiernan, "Long-term neuropathy after oxaliplatin treatment: challenging the dictum of reversibility," Oncologist, vol. 16, no. 5, pp. 708-716, 2011.

[13] E. Donzelli, M. Carfi, M. Miloso et al., "Neurotoxicity of platinum compounds: comparison of the effects of cisplatin and oxaliplatin on the human neuroblastoma cell line SH-SY5Y," Journal of Neuro-Oncology, vol. 67, no. 1-2, pp. 65-73, 2004.

[14] A. A. Argyriou, P. Polychronopoulos, G. Iconomou, E. Chroni, and H. P. Kalofonos, "A review on oxaliplatin-induced peripheral nerve damage," Cancer Treatment Reviews, vol. 34, no. 4, pp. 368-377, 2008.

[15] H.-W. Jung, J. W. Kim, J.-Y. Kim et al., "Effect of muscle mass on toxicity and survival in patients with colon cancer undergoing adjuvant chemotherapy," Supportive Care in Cancer, vol. 23, no. 3, pp. 687-694, 2015.

[16] D. L. Hershman, C. Lacchetti, R. H. Dworkin et al., "Prevention and management of chemotherapy-induced peripheral neuropathy in survivors of adult cancers: American society of clinical oncology clinical practice guideline," Journal of Clinical Oncology, vol. 32, no. 18, pp. 1941-1967, 2014.

[17] M. J. L. Bours, B. W. A. van der Linden, R. M. Winkels et al., "Candidate predictors of health-related quality of life of colorectal cancer survivors: a systematic review," Oncologist, vol. 21, no. 4, pp. 433-452, 2016.

[18] C. Brami, T. Bao, and G. Deng, "Natural products and complementary therapies for chemotherapy-induced peripheral neuropathy: a systematic review," Critical Reviews in Oncology/Hematology, vol. 98, pp. 325-334, 2016.

[19] S. O. D. Afonseca, F. M. Cruz, D. D. I. G. Cubero et al., "Vitamin E for prevention of oxaliplatin-induced peripheral neuropathy: a pilot randomized clinical trial," Sao Paulo Medical Journal, vol. 131, no. 1, pp. 35-38, 2013.

[20] L. A. Kottschade, J. A. Sloan, M. A. Mazurczak et al., "The use of vitamin $\mathrm{E}$ for the prevention of chemotherapy-induced peripheral neuropathy: results of a randomized phase III clinical trial," Supportive Care in Cancer, vol. 19, no. 11, pp. 1769-1777, 2011.

[21] Z. Salehi and M. Roayaei, "Effect of Vitamin E on oxaliplatininduced peripheral neuropathy prevention: a randomized controlled trial," International Journal of Preventive Medicine, vol. 6, no. 1, p. 104, 2015.

[22] C. Gedlicka, W. Scheithauer, B. Schüll, and G. V. Kornek, "Effective treatment of oxaliplatin-induced cumulative polyneuropathy with alpha-lipoic acid," Journal of Clinical Oncology, vol. 20, no. 15, pp. 3359-3361, 2002.

[23] Y. Guo, D. Jones, J. L. Palmer et al., "Oral alpha-lipoic acid to prevent chemotherapy-induced peripheral neuropathy: a randomized, double-blind, placebo-controlled trial," Supportive Care in Cancer, vol. 22, no. 5, pp. 1223-1231, 2014.

[24] P.-C. Lin, M.-Y. Lee, W.-S. Wang et al., "N-acetylcysteine has neuroprotective effects against oxaliplatin-based adjuvant chemotherapy in colon cancer patients: preliminary data," Supportive Care in Cancer, vol. 14, no. 5, pp. 484-487, 2006.

[25] T. Kono, N. Mamiya, N. Chisato et al., "Efficacy of Goshajinkigan for peripheral neurotoxicity of oxaliplatin in patients with advanced or recurrent colorectal cancer," Evidence-Based Complementary and Alternative Medicine, vol. 2011, Article ID 418481, 8 pages, 2011.

[26] T. Kono, T. Hata, S. Morita et al., "Goshajinkigan oxaliplatin neurotoxicity evaluation (GONE): a phase 2, multicenter, randomized, double-blind, placebo-controlled trial of goshajinkigan to prevent oxaliplatin-induced neuropathy," Cancer Chemotherapy and Pharmacology, vol. 72, no. 6, pp. 1283-1290, 2013. 
[27] M. Nishioka, M. Shimada, N. Kurita et al., "The Kampo medicine, Goshajinkigan, prevents neuropathy in patients treated by FOLFOX regimen," International Journal of Clinical Oncology, vol. 16, no. 4, pp. 322-327, 2011.

[28] E. Oki, Y. Emi, H. Kojima et al., "Preventive effect of Goshajinkigan on peripheral neurotoxicity of FOLFOX therapy (GENIUS trial): a placebo-controlled, double-blind, randomized phase III study," International Journal of Clinical Oncology, vol. 20, no. 4, pp. 767-775, 2015.

[29] N. Yoshida, T. Hosokawa, T. Ishikawa et al., "Efficacy of goshajinkigan for oxaliplatin-induced peripheral neuropathy in colorectal cancer patients," Journal of Oncology, vol. 2013, Article ID 139740, 8 pages, 2013.

[30] L. Yuan, G. Hui-juan, H. Jin-Chang, and W. Xiao-qin, "Clinical study of Jiawei Huangqi Guizhi Wuwu Decoction in preventing and treating peripheral neuro-sensory toxicity caused by oxaliplatin," Chinese Journal of Integrative Medicine, vol. 12, no. 1, pp. 19-23, 2006.

[31] Y. Liu, G. Zhu, L. Han, J. Liu, T. Ma, and H. Yu, "Clinical study on the prevention of oxaliplatin-induced neurotoxicity with guilongtongluofang: results of a randomized, double-blind, placebo-controlled trial," Evidence-Based Complementary and Alternative Medicine, vol. 2013, Article ID 541217, 7 pages, 2013.

[32] M. Ikeguchi, M. Yamamoto, Y. Arai et al., "Fucoidan reduces the toxicities of chemotherapy for patients with unresectable advanced or recurrent colorectal cancer," Oncology Letters, vol. 2, no. 2, pp. 319-322, 2011.

[33] W.-S. Wang, J.-K. Lin, T.-C. Lin et al., "Oral glutamine is effective for preventing oxaliplatin-induced neuropathy in colorectal cancer patients," Oncologist, vol. 12, no. 3, pp. 312-319, 2007.

[34] C. Tofthagen, C. Visovsky, J. Beckstead, I. Loy, and E. Eckelman, "Results of a strength and balance training pilot study for colorectal cancer survivors with peripheral neuropathy caused by oxaliplatin," Rehabilitation Oncology, vol. 32, no. 4, pp. 38-44, 2014.

[35] P. J. Coyne, W. Wan, P. Dodson, C. Swainey, and T. J. Smith, "A trial of scrambler therapy in the treatment of cancer pain syndromes and chronic chemotherapy-induced peripheral neuropathy," Journal of Pain and Palliative Care Pharmacotherapy, vol. 27, no. 4, pp. 359-364, 2013.

[36] K. Ogawa, M. Ogawa, K. Nishijima, M. Tsuda, and G. Nishimura, "Efficacy of contact needle therapy for chemotherapy-induced peripheral neuropathy," Evidence-Based Complementary and Alternative Medicine, vol. 2013, Article ID 928129, 5 pages, 2013.

[37] S. Schroeder, G. Meyer-Hamme, and S. Epplée, "Acupuncture for chemotherapy-induced peripheral neuropathy (CIPN): a pilot study using neurography," Acupuncture in Medicine, vol. 30, no. 1, pp. 4-7, 2012.

[38] B. Valentine-Davis and L. H. Altshuler, "Acupuncture for oxaliplatin chemotherapy-induced peripheral neuropathy in colon cancer: a retrospective case series," Medical Acupuncture, vol. 27, no. 3, pp. 216-223, 2015.

[39] S. Ushio, N. Egashira, H. Sada et al., "Goshajinkigan reduces oxaliplatin-induced peripheral neuropathy without affecting anti-tumour efficacy in rodents," European Journal of Cancer, vol. 48, no. 9, pp. 1407-1413, 2012.

[40] P. G. Clark, G. Cortese-Jimenez, and E. Cohen, "Effects of Reiki, yoga, or meditation on the physical and psychological symptoms of chemotherapy-induced peripheral neuropathy: a randomized pilot study," Journal of Evidence-Based Complementary and Alternative Medicine, vol. 17, no. 3, pp. 161-171, 2012.
[41] J. Marshall, A. Zakari, J. J. Hwang, V. Papadopoulos, A. Rosenberg, and C. Silver, "Ginkgo biloba (GB) extract as a neuroprotective agent in oxaliplatin ( $\mathrm{Ox})$-induced neuropathy," Journal of Clinical Oncology, vol. 14, supplement, p. 3670, 2004.

[42] G. Bianchi, G. Vitali, A. Caraceni et al., "Symptomatic and neurophysiological responses of paclitaxel- or cisplatin-induced neuropathy to oral acetyl-L-carnitine," European Journal of Cancer, vol. 41, no. 12, pp. 1746-1750, 2005.

[43] A. A. Argyriou, E. Chroni, A. Koutras et al., "A randomized controlled trial evaluating the efficacy and safety of vitamin E supplementation for protection against cisplatin-induced peripheral neuropathy: final results," Supportive Care in Cancer, vol. 14, no. 11, pp. 1134-1140, 2006.

[44] A. Pace, A. Savarese, M. Picardo et al., "Neuroprotective effect of vitamin E supplementation in patients treated with cisplatin chemotherapy," Journal of Clinical Oncology, vol. 21, no. 5, pp. 927-931, 2003.

[45] A. Pace, D. Giannarelli, E. Galiè et al., "Vitamin E neuroprotection for cisplatin neuropathy: a randomized, placebo-controlled trial," Neurology, vol. 74, no. 9, pp. 762-766, 2010.

[46] S. Cascinu, V. Catalano, L. Cordella et al., "Neuroprotective effect of reduced glutathione on oxaliplatin-based chemotherapy in advanced colorectal cancer: a randomized, double-blind, placebo-controlled trial," Journal of Clinical Oncology, vol. 20, no. 16, pp. 3478-3483, 2002.

[47] P. Milla, M. Airoldi, G. Weber, A. Drescher, U. Jaehde, and L. Cattel, "Administration of reduced glutathione in FOLFOX4 adjuvant treatment for colorectal cancer: effect on oxaliplatin pharmacokinetics, Pt-DNA adduct formation, and neurotoxicity," Anti-Cancer Drugs, vol. 20, no. 5, pp. 396-402, 2009.

[48] D. M. F. Savarese, G. Savy, L. Vahdat, P. E. Wischmeyer, and B. Corey, "Prevention of chemotheraphy and radiation toxicity with glutamine," Cancer Treatment Reviews, vol. 29, no. 6, pp. 501-513, 2003.

[49] S. Yoshida, A. Kaibara, N. Ishibashi, and K. Shirouzu, "Glutamine supplementation in cancer patients," Nutrition, vol. 17, no. 9, pp. 766-768, 2001.

[50] M. W. Saif, "Oral calcium ameliorating oxaliplatin-induced peripheral neuropathy," Journal of Applied Research, vol. 4, no. 4, pp. 576-582, 2004.

[51] L. Gamelin, M. Boisdron-Celle, R. Delva et al., "Prevention of oxaliplatin-related neurotoxicity by calcium and magnesium infusions: a retrospective study of 161 patients receiving oxaliplatin combined with 5-fluorouracil and leucovorin for advanced colorectal cancer," Clinical Cancer Research, vol. 10, no. 12, pp. 4055-4061, 2004.

[52] A. Grothey, D. A. Nikcevich, J. A. Sloan et al., "Intravenous calcium and magnesium for oxaliplatin-induced sensory neurotoxicity in adjuvant colon cancer: NCCTG N04C7," Journal of Clinical Oncology, vol. 29, no. 4, pp. 421-427, 2011.

[53] N. Knijn, J. Tol, M. Koopman et al., "The effect of prophylactic calcium and magnesium infusions on the incidence of neurotoxicity and clinical outcome of oxaliplatin-based systemic treatment in advanced colorectal cancer patients," European Journal of Cancer, vol. 47, no. 3, pp. 369-374, 2011.

[54] F. Wen, Y. Zhou, W. Wang et al., "Ca/Mg infusions for the prevention of oxaliplatinrelated neurotoxicity in patients with colorectal cancer: a meta-analysis," Annals of Oncology, vol. 24, no. 1, pp. 171-178, 2013.

[55] S. Balducci, G. Iacobellis, L. Parisi et al., "Exercise training can modify the natural history of diabetic peripheral neuropathy," 
Journal of Diabetes and Its Complications, vol. 20, no. 4, pp. 216223, 2006.

[56] J.-H. Park, J. Lee, M. Oh et al., "The effect of oncologists' exercise recommendations on the level of exercise and quality of life in survivors of breast and colorectal cancer: a randomized controlled trial," Cancer, vol. 121, no. 16, pp. 2740-2748, 2015.

[57] M. A. Pfeifer, D. R. Ross, J. P. Schrage et al., "A highly successful and novel model for treatment of chronic painful diabetic peripheral neuropathy," Diabetes Care, vol. 16, no. 8, pp. 11031115, 1993.

[58] C. Stevinson, H. Steed, W. Faught et al., "Physical activity in ovarian cancer survivors: associations with fatigue, sleep, and psychosocial functioning," International Journal of Gynecological Cancer, vol. 19, no. 1, pp. 73-78, 2009.

[59] H. Selagzi, B. Buyukakilli, B. Cimen, N. Yilmaz, and S. Erdogan, "Protective and therapeutic effects ot swimming exercise training on diabetic peripheral neuropathy of streptozotocininduced diabetic rats," Journal of Endocrinological Investigation, vol. 31, no. 11, pp. 971-978, 2008.

[60] B. B. Abuaisha, J. B. Costanzi, and A. J. M. Boulton, "Acupuncture for the treatment of chronic painful peripheral diabetic neuropathy: a long-term study," Diabetes Research and Clinical Practice, vol. 39, no. 2, pp. 115-121, 1998.

[61] K. D. Phillips, W. D. Skelton, and G. A. Hand, "Effect of acupuncture administered in a group setting on pain and subjective peripheral neuropathy in persons with human immunodeficiency virus disease," Journal of Alternative and Complementary Medicine, vol. 10, no. 3, pp. 449-455, 2004.

[62] Y. Tong, H. Guo, and B. Han, "Fifteen-day acupuncture treatment relieves diabetic peripheral neuropathy," Journal of Acupuncture and Meridian Studies, vol. 3, no. 2, pp. 95-103, 2010.

[63] C. Zhang, Y.-X. Ma, and Y. Yan, "Clinical effects of acupuncture for diabetic peripheral neuropathy," Journal of Traditional Chinese Medicine, vol. 30, no. 1, pp. 13-14, 2010.

[64] J. Guindon, L. Deng, B. Fan, J. Wager-Miller, and A. G. Hohmann, "Optimization of a cisplatin model of chemotherapy-induced peripheral neuropathy in mice: use of vitamin $\mathrm{C}$ and sodium bicarbonate pretreatments to reduce nephrotoxicity and improve animal health status," Molecular Pain, vol. 10, no. 1, article 56, 2014.

[65] S. Agthong, A. Kaewsema, and T. Charoensub, "Curcumin ameliorates functional and structural abnormalities in cisplatininduced neuropathy," Experimental Neurobiology, vol. 24, no. 2, pp. 139-145, 2015.

[66] M. S. Al Moundhri, S. Al-Salam, A. Al Mahrouqee, S. Beegam, and B. H. Ali, "The effect of curcumin on oxaliplatin and cisplatin neurotoxicity in rats: some behavioral, biochemical, and histopathological studies," Journal of Medical Toxicology, vol. 9, no. 1, pp. 25-33, 2013.

[67] M. I. Azevedo, A. F. Pereira, R. B. Nogueira et al., "The antioxidant effects of the flavonoids rutin and quercetin inhibit oxaliplatin-induced chronic painful peripheral neuropathy," Molecular Pain, vol. 9, no. 1, article 53, 2013.

[68] J. Ferrier, M. Bayet-Robert, B. Pereira et al., "A polyaminedeficient diet prevents oxaliplatin-induced acute cold and mechanical hypersensitivity in rats," PLoS ONE, vol. 8, no. 10, Article ID e77828, 2013. 


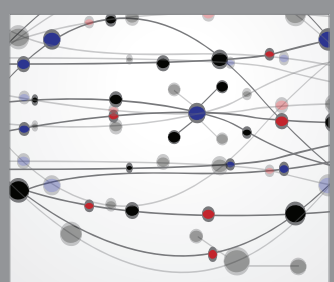

The Scientific World Journal
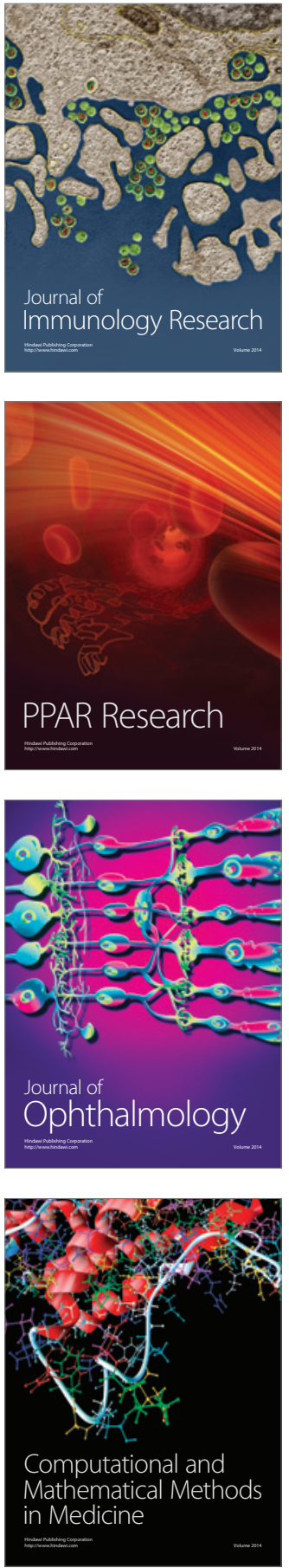

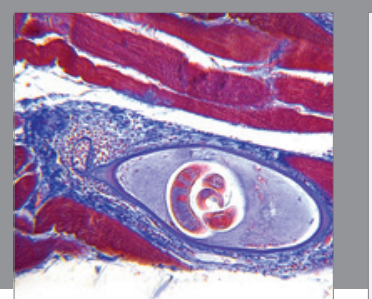

Gastroenterology Research and Practice
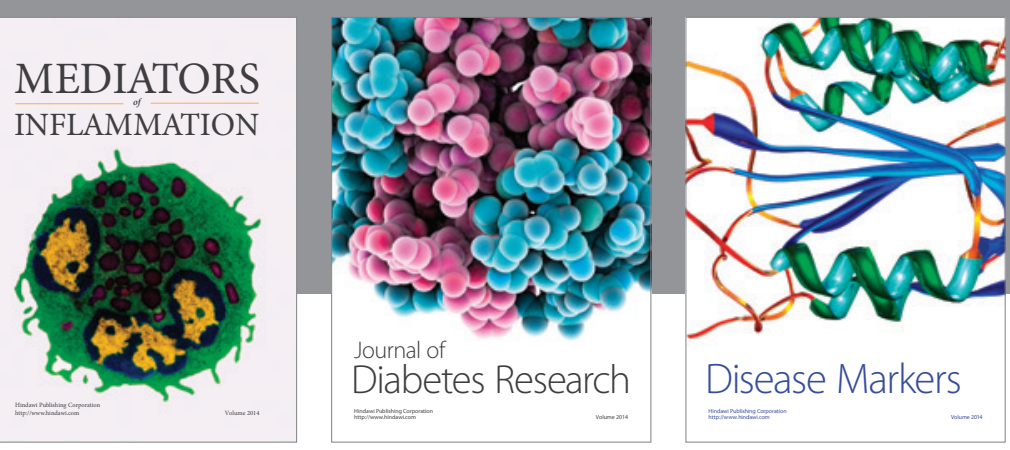

Disease Markers

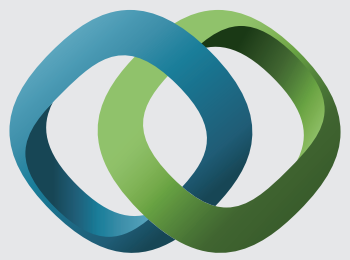

\section{Hindawi}

Submit your manuscripts at

https://www.hindawi.com
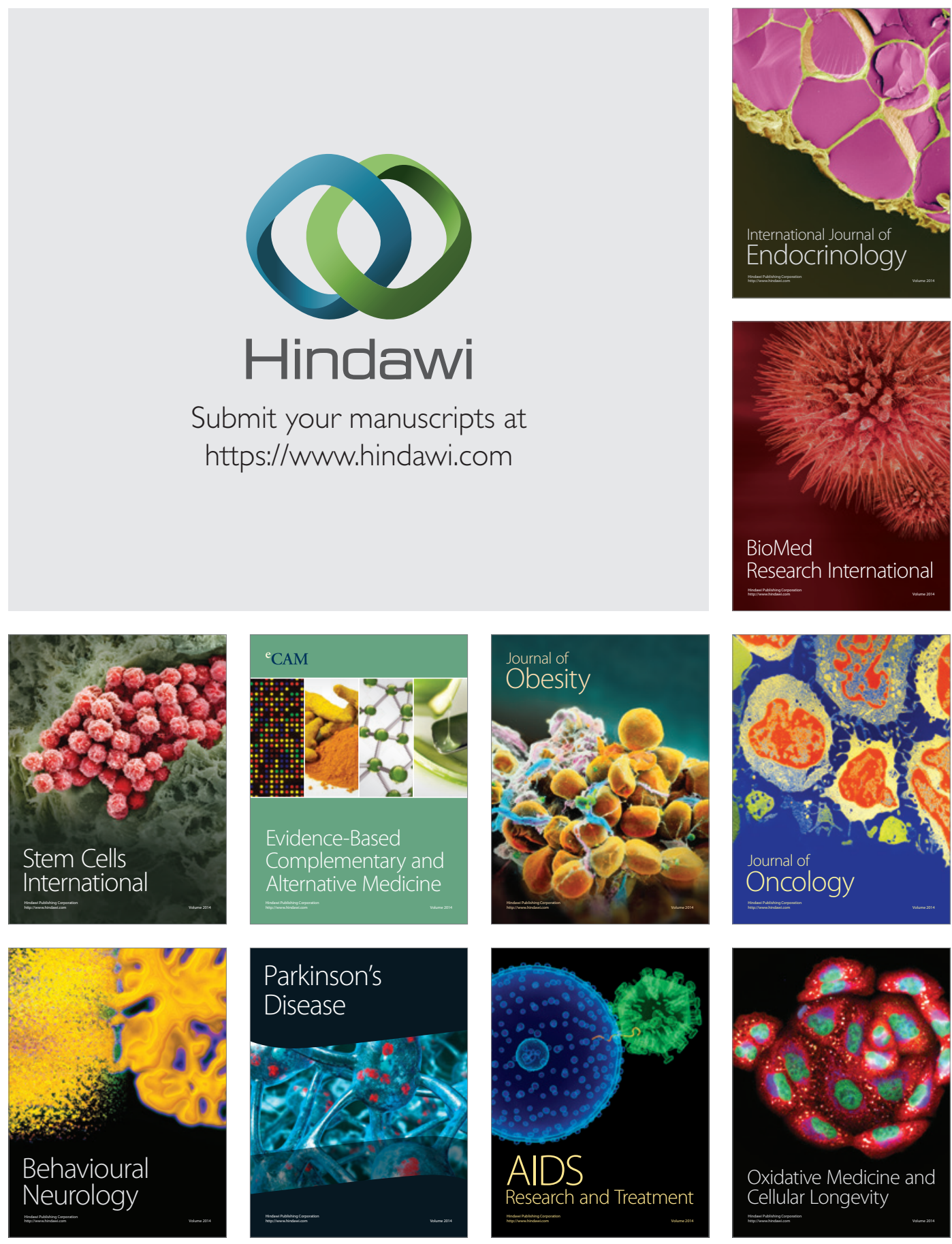